\title{
New Method for Measuring Angle-Resolved Phases in Photoemission
}

\author{
Daehyun You $\odot,{ }^{1}$ Kiyoshi Ueda, ${ }^{1, *}$ Elena V. Gryzlova $\odot,{ }^{2}$ Alexei N. Grum-Grzhimailo๑, ${ }^{2}$ Maria M. Popova $\odot,{ }^{2,3}$ \\ Ekaterina I. Staroselskaya, ${ }^{3}$ Oyunbileg Tugs, ${ }^{4}$ Yuki Orimo $\odot,{ }^{4}$ Takeshi Sato $\odot,{ }^{4,5,6}$ Kenichi L. Ishikawa $\odot,{ }^{4,5,6}$ \\ Paolo Antonio Carpeggiani®, ${ }^{7}$ Tamás Csizmadia, ${ }^{8}$ Miklós Füle, ${ }^{8}$ Giuseppe Sansone $\odot,{ }^{9}$ Praveen Kumar Maroju, ${ }^{9}$ \\ Alessandro D'Elia $\odot,{ }^{10,11}$ Tommaso Mazza, ${ }^{12}$ Michael Meyer $\odot,{ }^{12}$ Carlo Callegari $\odot,{ }^{13}$ Michele Di Fraia, ${ }^{13}$ \\ Oksana Plekan $\odot,{ }^{13}$ Robert Richter, ${ }^{13}$ Luca Giannessi, ${ }_{13}^{13}$ Enrico Allaria ${ }_{13}^{13}$ Giovanni De Ninno, ${ }^{13,15}$ Mauro Trovò, ${ }^{13}$ \\ Laura Badano, ${ }^{13}$ Bruno Diviacco $\odot{ }^{13}$ Giulio Gaio, ${ }^{13}$ David Gauthier, ${ }^{13, \$}$ Najmeh Mirian, ${ }^{13}$ Giuseppe Penco, ${ }^{13}$ \\ Primož Rebernik Ribič $\odot,{ }^{13,15}$ Simone Spampinati, ${ }^{13}$ Carlo Spezzani, ${ }^{13}$ and Kevin C. Prince ${ }^{13,16, \uparrow}$ \\ ${ }^{1}$ Institute of Multidisciplinary Research for Advanced Materials, \\ Tohoku University, Sendai 980-8577, Japan \\ ${ }^{2}$ Skobeltsyn Institute of Nuclear Physics, Lomonosov Moscow State University, \\ Moscow 119991, Russia \\ ${ }^{3}$ Faculty of Physics, Lomonosov Moscow State University, Moscow 119991, Russia \\ ${ }^{4}$ Department of Nuclear Engineering and Management, Graduate School of Engineering, \\ The University of Tokyo, 7-3-1 Hongo, Bunkyo-ku, Tokyo 113-8656, Japan \\ ${ }^{5}$ Photon Science Center, Graduate School of Engineering, The University of Tokyo, \\ 7-3-1 Hongo, Bunkyo-ku, Tokyo 113-8656, Japan \\ ${ }^{6}$ Research Institute for Photon Science and Laser Technology, The University of Tokyo, \\ 7-3-1 Hongo, Bunkyo-ku, Tokyo 113-0033, Japan \\ ${ }^{7}$ Institut für Photonik, Technische Universität Wien, 1040 Vienna, Austria \\ ${ }^{8}$ ELI-ALPS, ELI-HU Non-Profit Limited, Dugonics tér 13, H-6720 Szeged, Hungary \\ ${ }^{9}$ Physikalisches Institut, Albert-Ludwigs-Universität Freiburg, 79106 Freiburg, Germany \\ ${ }^{10}$ University of Trieste, Department of Physics, 34127 Trieste, Italy \\ ${ }^{11}$ IOM-CNR, Laboratorio Nazionale TASC, 34149 Basovizza, Trieste, Italy \\ ${ }^{12}$ European X-Ray Free Electron Laser Facility GmbH, Holzkoppel 4, 22869 Schenefeld, Germany \\ ${ }^{13}$ Elettra-Sincrotrone Trieste S.C.p.A., 34149 Basovizza, Trieste, Italy \\ ${ }^{14}$ INFN-Laboratori Nazionali di Frascati, 00044 Frascati, Rome, Italy \\ ${ }^{15}$ Laboratory of Quantum Optics, University of Nova Gorica, Nova Gorica 5001, Slovenia \\ ${ }^{16}$ Centre for Translational Atomaterials, Swinburne University of Technology, \\ 3122 Melbourne, Australia
}

(Received 7 January 2020; revised 15 June 2020; accepted 31 July 2020; published 30 September 2020)

\begin{abstract}
Quantum mechanically, photoionization can be fully described by the complex photoionization amplitudes that describe the transition between the ground state and the continuum state. Knowledge of the value of the phase of these amplitudes has been a central interest in photoionization studies and newly developing attosecond science, since the phase can reveal important information about phenomena such as electron correlation. We present a new attosecond-precision interferometric method of angle-resolved measurement for the phase of the photoionization amplitudes, using two phase-locked extreme ultraviolet pulses of frequency $\omega$ and $2 \omega$, from a free-electron laser. Phase differences $\Delta \tilde{\eta}$ between one- and twophoton ionization channels, averaged over multiple wave packets, are extracted for neon $2 p$ electrons as a function of the emission angle at photoelectron energies 7.9, 10.2, and $16.6 \mathrm{eV}$. $\Delta \tilde{\eta}$ is nearly constant for emission parallel to the electric vector but increases at $10.2 \mathrm{eV}$ for emission perpendicular to the electric vector. We model our observations with both perturbation and $a b$ initio theory and find excellent agreement. In the existing method for attosecond measurement, reconstruction of attosecond beating by interference of two-photon transitions (RABBITT), a phase difference between two-photon pathways
\end{abstract}

\footnotetext{
* Corresponding author.

kiyoshi.ueda@tohoku.ac.jp

Corresponding author. prince@elettra.eu

†Present address: LIDYL, CEA, CNRS, Université Paris-Saclay, CEA-Saclay, 91191 Gif-sur-Yvette, France.
}

Published by the American Physical Society under the terms of the Creative Commons Attribution 4.0 International license. Further distribution of this work must maintain attribution to the author(s) and the published article's title, journal citation, and DOI. 
involving absorption and emission of an infrared photon is extracted. Our method can be used for extraction of a phase difference between single-photon and two-photon pathways and provides a new tool for attosecond science, which is complementary to RABBITT.

\section{INTRODUCTION}

The age of attosecond physics was ushered in by the invention of methods for probing phenomena on a timescale less than femtoseconds [1]. A phenomenon occurring on this timescale is photoemission delay. When the photon energy is far from resonance, the photoemission delay for singlephoton ionization can be associated with the Wigner delay experienced by an electron scattering off the ionic potential [2]. Quantum mechanically, the photoionization process is fully described by the complex photoionization amplitudes describing transitions between the ground state and the continuum state. The photoemission delay can be expressed as the energy derivative of the phase of the photoionization amplitude, and, therefore, measuring the photoemission delay and the energy-dependent phase of the photoionization amplitude are practically equivalent. Their measurement is one of the central interests in attosecond science [3-14], because they are a fundamental probe of the photoionization process and can reveal important information about, for example, electron-electron correlations (see, e.g., Ref. [15]).

Currently, two methods are available to measure these quantities: streaking and reconstruction of attosecond beating by interference of two-photon transitions (RABBITT), both of which require the use of an IR dressing field. We present a new interferometric method of angle-resolved measurement for the photoionization phase, using two phase-locked extreme ultraviolet (XUV) pulses of frequency $\omega$ and $2 \omega$, from a free-electron laser (FEL), without a dressing field.

In attosecond streaking [16], an ultrafast, short-wavelength pulse ionizes an electron, and a femtosecond IR pulse acts as a streaking field, by changing the linear momentum of the photoelectron. In this technique, one can extract the photoemission delay difference between two photoemission lines at two different energies, arising, for example, from two different subshells [16] or the main line and satellites [15]. Generally, time-of-flight electron spectrometers located in the streaking direction (the direction of linear polarization) are used, so that this method does not give access to angular information. A related method is the attosecond clock technique [17-20], in which streaking by the circularly polarized laser pulse is in the angular direction.

The second technique for measuring photoemission delays, RABBITT, is interferometric: It uses a train of attosecond pulses dressed by a phase-locked IR pulse [21]. In the RABBITT technique, the phase difference between a pair of two-photon pathways whose final energy is separated by multiples of an infrared photon energy is extracted.
The extracted value is related to the phase difference of the two-photon ionization amplitudes at the pair of energies. For two energy points separated by twice the IR photon energy, the phase difference divided by twice the IR photon energy can be regarded as a finite difference approximation to the energy derivative of phase of the two-photon ionization amplitude. The pulse duration requirements are relaxed: For example, pulse trains and IR pulses of 30 fs duration may be used [6]. Usually, the IR pulse is the fundamental of the odd harmonics in the pulse train, although Loriot et al. [22] report a variant using the second harmonic. Recent work on phase retrieval includes methods based on photorecombination [13,23], two-color, two-photon ionization via a resonance [24], and a proposal to use successive harmonics of circularly polarized light [25].

The phase of the photoionization amplitude depends on photoelectron energy $\epsilon$, and it may also depend on the electron emission direction. There is a physical origin for the directional anisotropy of the amplitude: An electron wave packet may consist of two or more partial waves, with different angular momenta and phases. There has been significant theoretical work on the angle-dependent time delay, for example, Refs. [26-32], but fewer related experimental reports [12,28,33], all using the RABBITT technique. The Wigner delay is theoretically isotropic for single-photon ionization of $\mathrm{He}$, but Heuser et al. [28] observed an angular dependence in photoemission delay, attributed to the XUV + IR two-photon ionization process, inherent in RABBITT interferometry.

In the present work, we demonstrate interferometric measurements of the relative phase of single-photon and two-photon ionization amplitudes. The interference is created between a two-photon ionization process driven by a fundamental wavelength and a single-photon ionization process driven by its phase-locked, weaker, second harmonic, in a setup like that demonstrated at visible wavelengths [34]. Using short-wavelength, phase-locked XUV light, we measure angular distributions of photoelectrons emitted from neon and determine the phase difference for one- and two-photon ionization wave packets. The extremely short (attoseconds) pulses required for streaking or attosecond pulse trains for RABBITT are not needed, and, instead, access to the photoemission phase with attosecond precision is provided by optical phase control with a precision of a few attoseconds, which is available from the free-electron laser FERMI [35].

The rest of the manuscript is structured as follows: In Sec. II, we introduce the necessary notation and the basic 
processes that may be active in the experiment; in Secs. III and IV, we describe, respectively, the experimental and theoretical methods used. In Sec. V, we present and compare experimental and theoretical results. We discuss in Sec. VI the relationship between our data, namely, the angular distribution of photoelectrons created by collinearly polarized biharmonics, and the time-delay studies described in the introductory section. Section VII presents our summary and outlook, and the Appendix gives details of the derivation of some equations.

\section{NOTATION AND BASIC PROCESSES}

We use Hartree atomic units unless otherwise stated and spherical coordinates $\mathbf{r}=\{r, \theta, \varphi\}$ relative to the direction of polarization of the bichromatic field (linear horizontal in the experiment). We assume the electric dipole approximation, and the experiment is cylindrically symmetric about the electric vector, so that there is no dependence on the azimuthal angle $\varphi$. The bichromatic electric field is described by

$$
E(t)=\sqrt{I_{\omega}(t)} \cos \omega t+\sqrt{I_{2 \omega}(t)} \cos (2 \omega t-\phi),
$$

where $\omega$ and $2 \omega$ are angular frequencies, $I_{\omega}(t)$ and $I_{2 \omega}(t)$ are the pulse intensity envelopes, and $\phi$ denotes the $\omega-2 \omega$ relative phase.

We can consider the experimental sample as an ensemble of identical atoms of infinitesimal size, so we can reduce the theoretical treatment to that of a single atom centered at the coordinates' origin. The general form (omitting as implicit the dependence on $\theta, \varphi$ ) of an electron wave packet sufficiently far away from the origin is

$$
\int_{0}^{\infty} c(\epsilon) e^{-i \epsilon t} e^{i[\sqrt{2 \epsilon} r+f(r, \epsilon)+\eta(\epsilon)]} d \epsilon
$$

where $\epsilon$ is the photoelectron kinetic energy, $c(\epsilon)$ the realvalued amplitude, and $\eta(\epsilon)$ the phase and the term $f(r, \epsilon)=$ $(Z / \sqrt{2 \epsilon}) \ln \sqrt{8 \epsilon} r$ accounts for the Coulomb field of the residual ion with charge $Z$. In our case, $Z=+1$.

In the $\omega-2 \omega$ process, i.e., one driven by the field in Eq. (1), the wave packet can be expressed as

$$
\begin{aligned}
& \int_{0}^{\infty} e^{-i \epsilon t} e^{i[\sqrt{2 \epsilon} r+f(r, \epsilon)]} \\
& \quad \times\left\{c_{\omega}(\epsilon) e^{i \eta_{\omega}(\epsilon)}+c_{2 \omega}(\epsilon) e^{i\left[\eta_{2 \omega}(\epsilon)+\phi\right]}\right\} d \epsilon .
\end{aligned}
$$

The photoelectron yield as a function of optical phase $\phi$ (we omit the spatial coordinates on the right-hand side) is given by

$$
\begin{aligned}
I(\theta, \varphi ; \phi) \doteq & I(\theta ; \phi) \\
= & \int_{0}^{\infty}\left\{c_{\omega}(\epsilon)^{2}+c_{2 \omega}(\epsilon)^{2}+2 c_{\omega}(\epsilon) c_{2 \omega}(\epsilon)\right. \\
& \times \cos [\phi-\Delta \eta(\epsilon)]\} d \epsilon \\
\equiv & A_{0}+A \cos [\phi-\Delta \eta(\bar{\epsilon})],
\end{aligned}
$$

where $\bar{\epsilon}$ is the average kinetic energy of the wave packet and $\Delta \eta(\epsilon) \equiv \eta_{\omega}(\epsilon)-\eta_{2 \omega}(\epsilon)$ is the phase of the two-photon ionization relative to the single-photon ionization.

This treatment may be generalized to the case of multiple wave packets, that is to say, with more than one magnetic quantum number $m$ of the residual ion. Wave packets with each value of $m$ interfere separately and then incoherently add. In particular, expressing the photoionization yield as in Eq. (4)

$$
\begin{aligned}
I(\theta ; \phi) & =A_{0}+A \cos (\phi-\Delta \tilde{\eta}) \\
& =\sum_{m}\left[A_{0, m}+A_{m} \cos \left(\phi-\Delta \eta_{m}\right)\right],
\end{aligned}
$$

where summation is over the wave packets, leads to

$$
A_{0}=\sum_{m} A_{0, m}, \quad A e^{i \Delta \tilde{\eta}}=\sum_{m} A_{m} e^{i \Delta \eta_{m}} .
$$

The second equation defines an average phase difference $\Delta \tilde{\eta}$ of $\left\{\Delta \eta_{m}\right\}$, weighted in terms of the corresponding phase factors. Equations (4) and (5) indicate that the yield of photoelectrons emitted by a bichromatic pulse in a particular direction oscillates sinusoidally as a function of the optical phase $\phi$.

\section{EXPERIMENTAL METHODS AND SETUP}

The experimental methods are described elsewhere [35], and, here, we summarize the main aspects and the parameters used. The experiment is carried out at the low density matter beam line [36,37] of the FERMI freeelectron laser [38], using the velocity map imaging (VMI) spectrometer installed there. The VMI measures the projection of the photoelectron angular distribution (PAD) onto the planar detector (horizontal); the PAD is obtained as an inverse Abel transform of this projection, using the BASEX (basis set expansion) method [39]. The images are divided into two halves along the line of the electric vector, labeled "left" and "right," and analyzed separately. The PADs from the two halves agree generally, but the detector for the right half shows a small nonuniformity in detection efficiency. Therefore, the PADs are analyzed using the left half of the detector, denoted as $0^{\circ}-180^{\circ}$ below.

The sample consists of a mixture of helium and neon, and the helium PAD is used to calibrate the phase difference between the $\omega$ and $2 \omega$ fields. The atomic beam is produced 


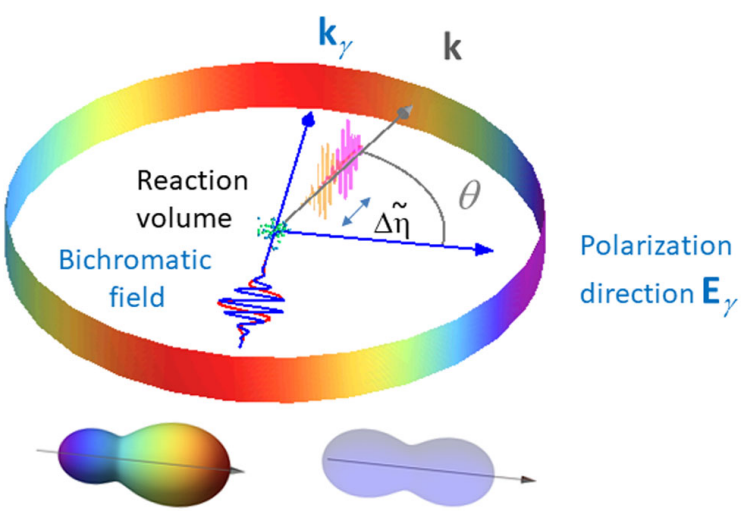

FIG. 1. Scheme of the experiment: Bichromatic, linearly polarized light (red and blue waves), with momentum $\mathbf{k}_{\gamma}$ and electric vector $\mathbf{E}_{\gamma}$, ionizes neon in the reaction volume. The electron wave packets (yellow and magenta waves) are emitted with electron momentum $\mathbf{k}$. The $m$-averaged phase difference $\Delta \tilde{\eta}$ between wave packets created by one- and two-photon ionization depends on the emission angle. The photoelectron angular distribution depends on the relative (optical) $\omega$-2 $\omega$ phase $\phi$. Lower figures: Polar plots of photoelectron intensity at $E_{k}=$ $16.6 \mathrm{eV}$ for coherent harmonics (asymmetric, colored plot) and incoherent harmonics (symmetric, gray plot).

by a supersonic expansion and defined by a conical skimmer and vertical slits. The length of the interaction volume along the light propagation direction is approximately $1 \mathrm{~mm}$. In other experiments [5,7], the use of two gases allows referencing of the photoemission delay of one electron to that of another. In the present case, we use the admixture of helium to provide a phase reference. When the free-electron laser wavelength is changed, the mechanical settings of the magnetic structures (undulators) creating the light are changed. This change may introduce an unknown phase error between fundamental and second harmonic light. We have recently shown that the PAD of helium $1 s$ electrons can be used to determine the absolute optical phase difference between the $\omega$ and $2 \omega$ fields, with an input of only a few theoretical parameters [40].

The light beam consists of two temporally overlapping harmonics with controlled relative phase $\phi$ [Eq. (1)] and irradiates the sample, as shown schematically in Fig. 1. The intense fundamental radiation causes two-photon ionization, while the weak second harmonic gives rise to singlephoton ionization. The energies of the photoelectrons created coherently in the two channels are identical, and electrons with the same linear momentum interfere [24]. The $\operatorname{PAD} I(\theta ; \phi)$ is measured as a function of the phase $\phi$; from the component oscillating with $\phi$, the scattering phases are extracted, as shown in Sec. V. The wavelength is then changed and the measurement repeated.

The relative phase of the two wavelengths is controlled by means of the electron delay line or phase shifter $[35,41]$ used previously. It has been calculated that the two pulses have good temporal overlap with slightly different durations and only a small mean variation of the relative phase of two wavelengths within the full width at half maximum of the pulses, for example, $0.07 \mathrm{rad}$ for a fundamental photon energy of $18.5 \mathrm{eV}$ [35].

The intensities of the two wavelengths for the experiments are set as follows. With the last undulator open (that is, inactive), the first five undulators are set to the chosen wavelength of the first harmonic. A small amount of spurious second harmonic radiation (intensity of the order of $1 \%$ of the fundamental) is produced by the undulators [42], and, to absorb this radiation, the gas filter available at FERMI is filled with helium. Helium is transparent at all of the fundamental wavelengths used in this study. The two-photon photoelectron signal from the neon and helium gas sample is observed with the VMI spectrometer. The last undulator is then closed to produce the second harmonic, and the photoelectron spectrum of the combined beams is observed. The single-photon ionization by the second harmonic is at least an order of magnitude stronger than the two-photon ionization by the fundamental. The helium gas pressure in the gas filter is then adjusted to achieve a ratio of the ionization rates due to two-photon and single-photon ionization of $1: 2$ for kinetic energies of 7.0 and $10.2 \mathrm{eV}$. For the kinetic energy of $15.9 \mathrm{eV}$, the ratio is set to $1: 4$. The bichromatic beam is focused by adjusting the curvature of the Kirkpatrick-Baez active optics [43] and verified experimentally by measuring the focal spot size of the second harmonic with a Hartmann wavefront sensor. This instrument is not able to measure the spot size of the beams at the fundamental wavelengths, so it is calculated [44]. The measured spot is elliptical with a size of $(4.5 \pm 1) \times(6.5 \pm 1) \mu \mathrm{m}^{2}(\mathrm{FWHM})$, and the estimated pulse duration is $100 \mathrm{fs}$.

Table I summarizes the experimental parameters: fundamental photon energy $(\hbar \omega)$, kinetic energy $\left(E_{k}=\right.$ $2 \hbar \omega-21.6 \mathrm{eV})$ of the $\mathrm{Ne}$ photoelectrons emitted via

TABLE I. Experimental parameters. $E_{k}$ is the kinetic energy of the Ne $2 p$ photoelectrons. The pulse energies at the sample are calculated by multiplying the values at the source by the transmission of the beam line [37], which takes account of reflection and geometric losses. Pulse energies and irradiance are those of the fundamental.

\begin{tabular}{lccccc}
\hline \hline$\hbar \omega, \mathrm{eV}$ & $E_{k}, \mathrm{eV}$ & $\begin{array}{c}\text { Pulse energy, } \mu \mathrm{J} \\
\text { (at source) }\end{array}$ & $\begin{array}{c}\text { Beam line } \\
\text { Transmission }\end{array}$ & $\begin{array}{c}\text { Pulse energy, } \mu \mathrm{J} \\
\text { (at sample) }\end{array}$ & $\begin{array}{c}\text { Average irradiance } \\
\mathrm{W} / \mathrm{cm}^{2}\end{array}$ \\
\hline 14.3 & 7.0 & 45 & 0.10 & 4.5 & $2.3 \times 10^{14}$ \\
15.9 & 10.2 & 95 & 0.13 & 12.4 & $6.2 \times 10^{14}$ \\
19.1 & 16.6 & 84 & 0.23 & 19.3 & $9.5 \times 10^{14}$ \\
\hline \hline
\end{tabular}


single-photon $(2 \omega)$ or two-photon $(\omega+\omega)$ ionization, average pulse energy of the first harmonic at the source and at the sample, beam line transmission, and average irradiance at the sample calculated from the above spot sizes and pulse durations. The estimate of the pulse energy at $\hbar \omega=14.3 \mathrm{eV}$ is indirect, since the FERMI intensity monitors do not function at this energy, because they are based on ionization of nitrogen gas, and the photon energy is below the threshold for ionization. The method employed is to first use the in-line spectrometer to measure spectra at $15.9 \mathrm{eV}$ energy and, simultaneously, the pulse energies from the gas cell monitors, which gives a calibration of the spectrometer intensity versus pulse energy at this wavelength. Then, spectrometer spectra are measured at $14.3 \mathrm{eV}$, and corrected for grating efficiency and detector sensitivity, to yield pulse energies.

\section{THEORY}

We now consider the physics of the experiment from two theoretical points of view: real-time $a b$ initio simulations, which are very accurate but computationally expensive, and perturbation theory, which allows us to explore the physics analytically and gain insights with relatively low computational costs.

\section{A. Real-time $a b$ initio simulations}

We numerically compute the photoionization of $\mathrm{Ne}$ irradiated by two-color XUV pulses, using the time-dependent complete-active-space self-consistent field (TD-CASSCF) method $[45,46]$ and the parameters in Table II. The pulse length is chosen to be $10 \mathrm{fs}$ for reasons of computational economy. It has been shown that the pulse length does not affect the result, provided the photoionization is nonresonant, i.e., no resonances occur within the photon bandwidth $[47,48]$. As a further check, we also calculate the phase shift difference at $14.3 \mathrm{eV}$ photon energy for pulse durations of 5, 10, and 20 fs and find identical results. Thus, we can safely scale the results to the present longer experimental pulses.

Neither the absolute intensity nor the ratio of intensities of the harmonics influences the calculated phase, as we show below. The dynamics of the laser-driven multielectron system is described by the time-dependent Schrödinger equation (TDSE):

TABLE II. Parameters used for theoretical calculations. $I_{\omega}$ and $I_{2 \omega}$ are the peak intensities of the fundamental and second harmonic, respectively.

\begin{tabular}{lcc}
\hline \hline$\hbar \omega, \mathrm{eV}$ & $I_{\omega}, \mathrm{W} / \mathrm{cm}^{2}$ & $I_{2 \omega}, \mathrm{W} / \mathrm{cm}^{2}$ \\
\hline 14.3 & $1 \times 10^{13}$ & $2.32 \times 10^{8}$ \\
15.9 & $1 \times 10^{13}$ & $1.18 \times 10^{8}$ \\
19.1 & $1 \times 10^{13}$ & $2.82 \times 10^{8}$ \\
\hline \hline
\end{tabular}

$$
i \frac{\partial \Psi(t)}{\partial t}=\hat{H}(t) \Psi(t)
$$

where the time-dependent Hamiltonian is

$$
\hat{H}(t)=\hat{H}_{1}(t)+\hat{H}_{2},
$$

with the one-electron part

$$
\hat{H}_{1}(t)=\sum_{i=1}^{Z} \hat{h}\left(\mathbf{r}_{i}, t\right)
$$

and the two-electron part

$$
\hat{H}_{2}=\sum_{i=1}^{Z} \sum_{j<i} \frac{1}{\left|\mathbf{r}_{i}-\mathbf{r}_{j}\right|} .
$$

We employ the velocity gauge for the laser-electron interaction in the one-body Hamiltonian:

$$
\hat{h}(\mathbf{r}, t)=\frac{\mathbf{k}^{2}}{2}+\mathbf{A}(t) \cdot \mathbf{k}-\frac{Z}{|\mathbf{r}|},
$$

where $\mathbf{A}(t)=-\int \mathbf{E}(t) d t$ is the vector potential, $\mathbf{E}(t)$ is the laser electric field [see Eq. (1)], and $Z(=10$ for $\mathrm{Ne})$ the atomic number.

In the TD-CASSCF method, the total electronic wave function is given in the configuration interaction (CI) expansion:

$\Psi\left(x_{1}, x_{2}, \ldots, x_{N}, t\right)=\sum_{I} C_{I}(t) \Phi_{I}\left(x_{1}, x_{2}, \ldots, x_{N}, t\right)$,

where $x_{n}=\left\{\mathbf{r}_{n}, \sigma_{n}\right\}$ is the joint designation for spatial and spin coordinates of the $n$th electron. The electronic configuration $\Phi_{I}\left(x_{1}, x_{2}, \ldots, x_{N}, t\right)$ is a Slater determinant composed of spin orbital functions $\left\{\psi_{p}(\mathbf{r}, t) \times s(\sigma)\right\}$, where $\left\{\psi_{p}(\mathbf{r}, t)\right\}$ and $\{s(\sigma)\}$ denote spatial orbitals and spin functions, respectively. Both the CI coefficients $\left\{C_{I}\right\}$ and orbitals vary in time.

The TD-CASSCF method classifies the spatial orbitals into three groups: doubly occupied and time-independent frozen core (FC), doubly occupied and time-dependent dynamical core (DC), and fully correlated active orbitals:

$$
\Psi=\hat{A}\left[\Phi_{\mathrm{fc}} \Phi_{\mathrm{dc}} \sum_{I} \Phi_{I} C_{I}\right],
$$

where $\hat{A}$ denotes the antisymmetrization operator, $\Phi_{\mathrm{fc}}$ and $\Phi_{\mathrm{dc}}$ the closed-shell determinants formed with numbers $n_{\mathrm{fc}}$ FC orbitals and $n_{\mathrm{dc}}$ DC orbitals, respectively, and $\left\{\Phi_{I}\right\}$ the determinants constructed from $n_{a}$ active orbitals. We consider all the possible distributions of active electrons among active orbitals. Thanks to this decomposition, we 
can significantly reduce the computational cost without sacrificing the accuracy in the description of correlated multielectron dynamics. The equations of motion that describe the temporal evolution of the CI coefficients $\left\{C_{I}\right\}$ and the orbital functions $\left\{\psi_{p}\right\}$ are derived by use of the time-dependent variational principle [45]. The numerical implementation of the TD-CASSCF method for atoms is detailed in Refs. [46,49].

\section{B. Extraction of the photoelectron angular distribution and the phase shift difference}

From the obtained time-dependent wave functions, we extract the angle-resolved photoelectron energy spectrum (ARPES) by use of the time-dependent surface flux (tSURFF) method [50]. This method computes the ARPES from the electron flux through a surface located at a certain radius $R_{s}$, beyond which the outgoing flux is absorbed by the infinite-range exterior complex scaling [49,51].

We introduce the time-dependent momentum amplitude $a_{p}(\boldsymbol{k}, t)$ of orbital $p$ for photoelectron momentum $\mathbf{k}$, defined by

$$
\begin{aligned}
a_{p}(\mathbf{k}, t) & =\left\langle\chi_{\mathbf{k}}(\mathbf{r}, t)\left|u\left(R_{s}\right)\right| \psi_{p}(\mathbf{r}, t)\right\rangle \\
& \equiv \int_{r>R_{s}} \chi_{\mathbf{k}}^{*}(\mathbf{r}, t) \psi_{p}(\mathbf{r}, t) d^{3} \mathbf{r},
\end{aligned}
$$

where $\chi_{\mathbf{k}}(\mathbf{r}, t)$ denotes the Volkov wave function and $u\left(R_{s}\right)$ the Heaviside function which is unity for $r>R_{s}$ and vanishes otherwise. The use of the Volkov wave function implies that we neglect the effects of the Coulomb force from the nucleus and the other electrons on the photoelectron dynamics outside $R_{s}$, which is confirmed to be a good approximation [52]. The photoelectron momentum distribution $\rho(\mathbf{k})$ is given by

$$
\rho(\mathbf{k})=\sum_{p q} a_{p}(\mathbf{k}, \infty) a_{q}^{*}(\mathbf{k}, \infty)\left\langle\Psi(t)\left|\hat{E}_{p}^{q}\right| \Psi(t)\right\rangle,
$$

with $\hat{E}_{p}^{q} \equiv \sum_{\sigma} \hat{a}_{q \sigma}^{\dagger} \hat{a}_{p \sigma}$. One obtains $a_{p}(\mathbf{k}, \infty)$ by numerically integrating:

$$
\begin{aligned}
-i \frac{\partial}{\partial t} a_{p}(\mathbf{k}, t)= & \left\langle\chi_{\mathbf{k}}(t)\left|\left[\hat{h}_{s}, u\left(R_{s}\right)\right]\right| \psi_{p}(t)\right\rangle \\
& +\sum_{q} a_{q}(\mathbf{k}, t)\left\{\left\langle\psi_{q}(t)|\hat{F}| \psi_{p}(t)\right\rangle-R_{p}^{q}\right\},
\end{aligned}
$$

where $\hat{h}_{s}=\mathbf{k}^{2} / 2+\mathbf{A}(t) \cdot \mathbf{k}, R_{p}^{q}=i\left\langle\psi_{q} \mid \dot{\psi}_{p}\right\rangle-\left\langle\psi_{q}|\hat{h}| \psi_{p}\right\rangle$ and $\hat{F}$ denotes a nonlocal operator describing the contribution from the interelectronic Coulomb interaction $[46,49]$. The numerical implementation of tSURFF to TD-CASSCF is detailed in Ref. [52].

We evaluate the photoelectron angular distribution $I(\theta ; \phi)$ as a slice of $\rho(\mathbf{k})$ at the value of $|\mathbf{k}|$ corresponding to the photoelectron peak and as a function of the optical phase $\phi$. Then, employing a fitting procedure very similar to that used for the experimental data, we extract the phase shift difference $\Delta \tilde{\eta}$ between single-photon and two-photon ionization at photoelectron energies 7.0, 10.2, and $16.6 \mathrm{eV}$. The results are shown in Fig. 2.

\section{Perturbation theory}

In the experiment, the number of optical cycles in the pulse is of the order of 400 for the fundamental, and, therefore, we can treat the field as having constant amplitude and omit the initial phase of the field with respect to the envelope (carrier-envelope phase). Within the perturbation theory, we check that our final results with an envelope including 100 optical cycles or more differ only within the optical linewidth from those obtained with the constant amplitude field. The bichromatic electric field is then described by Eq. (1), with time-independent $I_{\omega}$ and $I_{2 \omega}$. The calculations described below are carried out for 384 optical cycles and a peak intensity of $1 \times 10^{12} \mathrm{~W} / \mathrm{cm}^{2}$. However, neither the absolute intensity nor the ratio of intensities of the harmonics influences the calculated phase, as we show below.

We make two main assumptions: the dipole approximation for the interaction of the atom with the classically described electromagnetic field and the validity of the lowest nonvanishing order perturbation theory with respect to this interaction. These approximations are well fulfilled for neon in the FEL spectral range and intensities of interest here. We expand the amplitudes in the lowest nonvanishing order of perturbation theory in terms of matrix elements of the operator of evolution [53]. The expansion implies that, in the second-order amplitude, all virtual intermediate states are taken into account. Excitations of the seven lowest and most important intermediate dipole-allowed states originating from configurations $\left(2 p^{5} 3 s, 4 s, 3 d\right)$ are accounted for accurately within the multiconfiguration intermediate-coupling approximation with relativistic Breit-Pauli corrections in the atomic Hamiltonian. All other virtual states (of infinite number), including those in the continuum, are accounted for by a variationally stable method [54,55] in the Hartree-Fock-Slater approximation. More details can be found in Ref. [56]. Further derivations within the independent particle approximation are given in the Appendix.

\section{RESULTS}

We extract $\Delta \tilde{\eta}(\theta)$ from the measured PADs at three combinations of $\omega$ and $2 \omega$ (corresponding to photoelectron kinetic energies of 7.0, 10.2, and $16.6 \mathrm{eV}$ ), at each $5^{\circ}$ interval of the polar angle. The spatial and temporal symmetry properties of the system impose constraints on the oscillatory behavior of the two emission hemispheres. Upon reflection in a plane perpendicular to the electric vector $(\theta \rightarrow \pi-\theta)$, the electric field defined in Eq. (1) is 


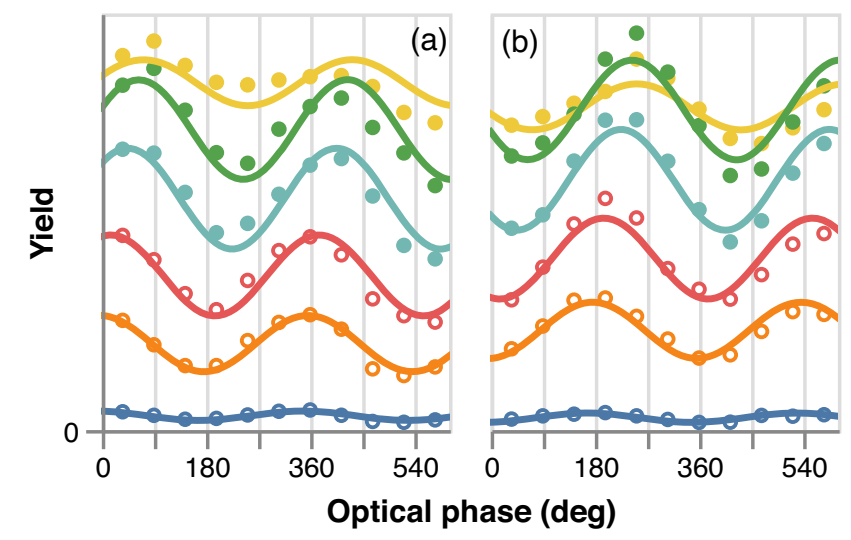

\section{Polar angle (deg)}

Panel A / B

75-80 / 100-105

60-65 / 115-120

45-50 / 130-135

30-35 / 145-150

15-20 / 160-165

0-5 / 175-180
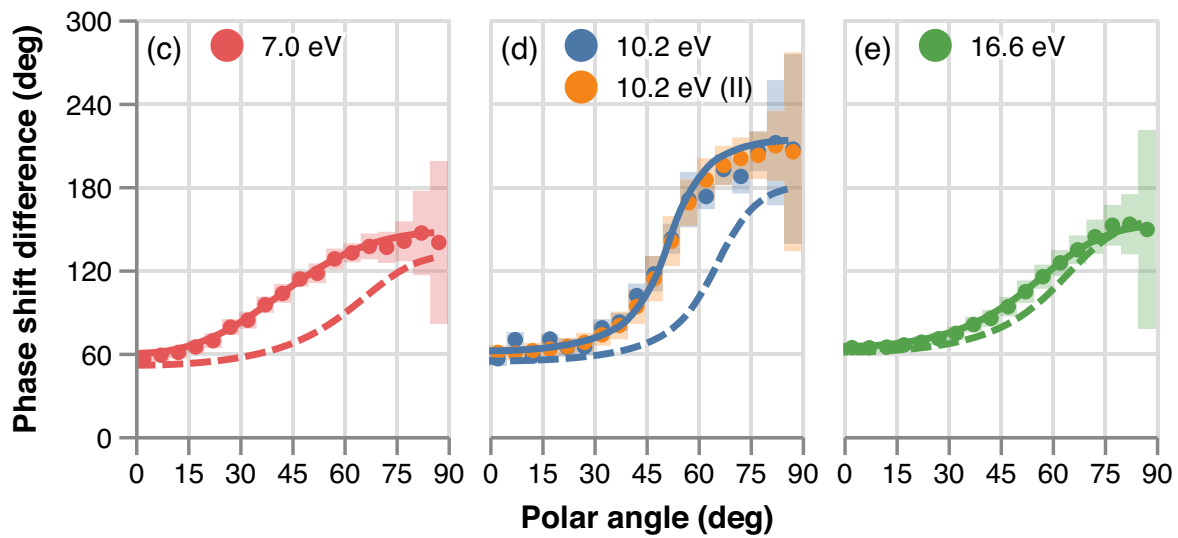

FIG. 2. Upper: Typical photoelectron yields $I(\theta ; \phi)$ as a function of optical phase $\phi$ at intervals of polar angles $\theta$. The signal is integrated over the $5^{\circ}$ intervals shown on the right. The curves are not shifted; thus, their vertical position reflects directly the value $A_{0}$ in Eq. (4). The photoelectron kinetic energy is $7.0 \mathrm{eV}$. Circles are experimental results; lines are sinusoidal fits of the experimental results. Lower: Extracted phase shift differences as a function of the polar angles, for four datasets and three photoelectron kinetic energies: left (c), $7.0 \mathrm{eV}$; middle (d), $10.2 \mathrm{eV}$; right (e), $16.6 \mathrm{eV}$. Circles are experimental results; shaded areas show their uncertainties. Dashed lines, perturbation theory; solid lines, real-time ab initio theory. Note that the curves in (a) and (b) oscillate in antiphase, because they correspond to emission directions on opposite sides of the photon propagation direction.

inverted: $E(t) \rightarrow-E(t)$, and the $\omega-2 \omega$ relative phase becomes $\phi+\pi$. From the arguments above, Eq. (5) becomes

$$
\begin{aligned}
I(\pi-\theta, \phi+\pi) & =A_{0}+A \cos [\phi+\pi-\Delta \tilde{\eta}(\pi-\theta)] \\
& =A_{0}-A \cos [\phi-\Delta \tilde{\eta}(\pi-\theta)],
\end{aligned}
$$

where we omit the argument $\bar{\epsilon}$ and include explicitly the argument $\theta$. Comparison with Eq. (4) indicates that the intensities at the two opposite angles oscillate in antiphase, that is, $\Delta \tilde{\eta}(\pi-\theta)=\Delta \tilde{\eta}(\theta)+\pi$. It can be seen in Figs. 2(a) and 2(b) that the experimental data do indeed oscillate in antiphase for each angular interval over $\theta$. Since this is a symmetry constraint, it is imposed in the analysis of the data.

In Figs. 2(c)-2(e), it can be seen that there is a significant increase of $\Delta \tilde{\eta}(\theta)$ at approximately $90^{\circ}$, especially for $10.2 \mathrm{eV}$. The angular-dependent variations of $\Delta \tilde{\eta}(\theta)$ at 7.0 and $16.6 \mathrm{eV}$ are similar. We perform calculations for the phase shift differences $\Delta \tilde{\eta}(\theta)$ using both perturbation theory and real-time $a b$ initio methods (see Sec. IV and the Appendix), and both theories reproduce well the observed behavior; see Figs. 2(c)-2(e). The perturbation theory result at the intermediate angles is very sensitive to the contribution of the $p-d-f$ two-photon ionization path, which may not be accurately reproduced by the localpotential approximation in summation over the Rydberg and continuum $d$ states.

Figure 3 shows the theoretical dependence of $\Delta \tilde{\eta}(\theta)$ on electron kinetic energy and polar angle $\theta$, calculated using perturbation theory. There is a single-photon $2 p \rightarrow 3 s$ resonance of the fundamental wavelength at $16.7 \mathrm{eV}$ photon energy (12 eV kinetic energy for the two-photon or second harmonic). The behavior of $\Delta \tilde{\eta}$ in the region of the resonance is complicated: We can clearly see that $\Delta \tilde{\eta}(\theta)$ at $\theta \sim 90^{\circ}$ increases near the resonance around $12 \mathrm{eV}$ and then returns to a value similar to that at approximately $7 \mathrm{eV}$. This result indicates that the large phase shift difference observed at $10.2 \mathrm{eV}$ in Fig. 2(d) is due to the influence of the resonance at $12.0 \mathrm{eV}[32,33]$ and suggests that future 


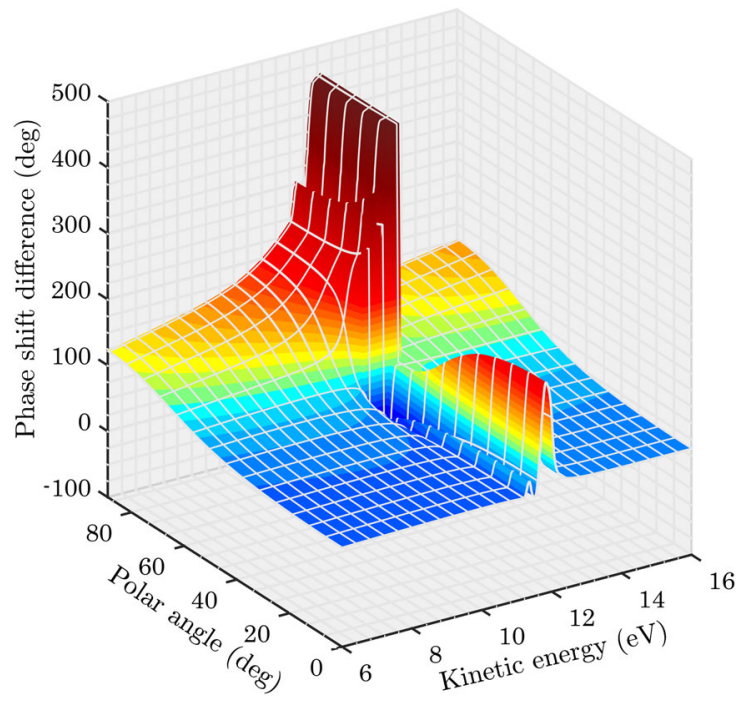

FIG. 3. Phase shift differences $\Delta \tilde{\eta}$ of two-photon ionization relative to single-photon ionization, calculated by perturbation theory, as a function of the polar angle $\theta$ and photoelectron kinetic energy. The large variation near $12.0 \mathrm{eV}$ kinetic energy is due to the $2 p \rightarrow 3 s$ resonance.

experiments should explore this region in fine detail, to observe the predicted rapid changes in $\Delta \tilde{\eta}$. Both theories reproduce this behavior well, with the time-dependent $a b$ initio method exhibiting excellent agreement, validating the present experimental method.

We show in the Appendix that the method is independent of the relative intensities of the fundamental and second harmonic radiation; see Eqs. (A5)-(A9). This advantage is considerable from an experimental point of view, as it is not necessary to measure precisely the intensity and focal spot shape. Furthermore, there are no effects due to volume averaging over the Gaussian spot profile or over the duration of the pulses. We verify this conclusion experimentally for the kinetic energy of $16.6 \mathrm{eV}$ [Fig. 2(d)], where the ratio of ionization rates is $1: 4$ (rather than $1: 2$ used for the other energies), and the experiment and theory agree well.

\section{DISCUSSION}

In this section, we elucidate the relationship of our data, i.e., photoelectron angular distributions created by collinearly polarized biharmonics, to time-delay studies described in the introduction. We limit ourselves to the case where any discrete state in the continuum (autoionizing state) or in the discrete spectrum lies outside the bandwidth of the pulses $(<0.02 \mathrm{eV}$ in the present case). These conditions are well fulfilled in our experiments. The resonant case is discussed elsewhere [10].

We first consider the simple situation of photoionization from a spherically symmetric orbital $s$. The present method can be extended straightforwardly to inner shell ionization of atoms, such as $1 s^{2}$ of Ne. Single-photon ionization leads to a continuum state with angular momentum $p$, while twophoton ionization leads to two final quantum states $s$ and $d$. Then, the $\operatorname{PAD} I_{e}(\theta)$ is described by

$$
\begin{aligned}
I_{e}(\theta)= & \mid c_{s} e^{i \eta_{s}} Y_{00}(\theta, \varphi)+c_{p} e^{i\left(\eta_{p}+\phi\right)} Y_{10}(\theta, \varphi) \\
& +\left.c_{d} e^{i \eta_{d}} Y_{20}(\theta, \varphi)\right|^{2},
\end{aligned}
$$

where $c_{s}, c_{p}$, and $c_{d}$ are real-valued partial-wave amplitudes and $\eta_{s}, \eta_{p}$, and $\eta_{d}$ are the corresponding arguments. $I_{e}(\theta)$ can also be expressed as

$$
I_{e}(\theta)=\left(c_{s}^{2}+c_{p}^{2}+c_{d}^{2}\right)\left[1+\sum_{l=1}^{4} \beta_{l} P_{l}(\cos \theta)\right],
$$

where $P_{l}(\cos \theta)$ are the Legendre polynomials describing the angular distributions and $\beta_{l}$ are the corresponding asymmetry parameters. After some algebra, we have [40]

$$
\begin{gathered}
\beta_{3}=\frac{6 \sqrt{15} c_{d} c_{p} \cos \left(-\eta_{d}+\eta_{p}+\phi\right)}{5\left(c_{d}^{2}+c_{p}^{2}+c_{s}^{2}\right)} \\
\equiv\left[\beta_{3}\right]_{0} \cos \left[\phi-\left(\eta_{d}-\eta_{p}\right)\right], \\
\beta_{1}-\frac{2}{3} \beta_{3}=\frac{2 \sqrt{3} c_{p} c_{s} \cos \left(\eta_{p}-\eta_{s}+\phi\right)}{c_{d}^{2}+c_{p}^{2}+c_{s}^{2}} \\
\equiv\left[\beta_{1}-\frac{2}{3} \beta_{3}\right]_{0} \cos \left[\phi-\left(\eta_{s}-\eta_{p}\right)\right],
\end{gathered}
$$

where $\left[\beta_{3}\right]_{0}$ and $\left[\beta_{1}-\frac{2}{3} \beta_{3}\right]_{0}$ are constants. Thus, if we record PADs as a function of $\phi$ and extract $\beta_{l}(l=1-4)$, we can directly read off $\eta_{d}-\eta_{p}$ and $\eta_{s}-\eta_{p}$ from the oscillations of $\beta_{3}$ and $\beta_{1}-\frac{2}{3} \beta_{3}$ using Eqs. (20) and (21). Let us recall that the Wigner delay of each partial wave, $\tau_{l}$, corresponds to the energy derivative of the argument of the amplitude [note that $Y_{l 0}(\theta, \varphi)$ are real], $\tau_{l}(\epsilon)=$ $\left(d \eta_{l}(\epsilon) / d \epsilon\right)$ [2]. By measuring $\eta_{d}-\eta_{p}$ and $\eta_{s}-\eta_{p}$ as a function of the energy, one can take the energy derivative and obtain the Wigner delay differences $\tau_{l}(\epsilon)-\tau_{p}(\epsilon)$ with $l=s$ and $d$. In simple models, like the Hartree-Fock approximation, $\left(d \eta_{l}(\epsilon) / d \epsilon\right)=\left(d \delta_{l}(\epsilon) / d \epsilon\right)$, where $\delta_{l}(\epsilon)$ is the scattering phase, while in more complicated cases, an extra energy-dependent phase may be acquired by the partial amplitude [27].

We now group the $s$ and $d$ waves as a two-photonionization wave packet. Then, the photoelectron wave packet in a given direction $\theta$ sufficiently far from the nucleus and the corresponding PAD are expressed as Eqs. (3) and (4), respectively. The energy derivative of $\Delta \eta(\theta) \equiv \eta_{\omega}(\theta)-\eta_{2 \omega}(\theta)$ is a difference between the group delays of the two wave packets, generated by twoand single-photon ionization, respectively. In the original photoemission delay experiment [16] with attosecond 
streaking, for example, Ne $2 s$ and $2 p$ electrons are ionized by an attosecond pulse to different final kinetic energies. As a result, the more energetic photoelectron from $2 p$ arrives at the detector much earlier than that from $2 s$, regardless of the measured delay. The situation is similar for subsequent measurements using streaking and RABBITT. In great contrast, in the present case, both single- and two-photon ionization result in the same photoelectron energy. Therefore, the single- and twophoton-ionization wave packets actually reach a given distance with a relative (group) delay given by $\partial \Delta \eta / \partial \epsilon$.

By comparing Eqs. (4) and (18), we can describe the phase factor $e^{i \Delta \eta(\theta)}$ with $\Delta \eta(\theta) \equiv \eta_{\omega}(\theta)-\eta_{2 \omega}(\theta)$ being the angle-resolved phase difference between the two-photon and single-photon ionization amplitudes as

$$
\begin{aligned}
& A(\theta) e^{i \Delta \eta(\theta)} \\
& \quad=2 c_{p} Y_{10}(\theta, \varphi)\left[c_{d} Y_{20}(\theta, \varphi) e^{i\left(\eta_{d}-\eta_{p}\right)}+c_{s} Y_{00}(\theta) e^{i\left(\eta_{s}-\eta_{p}\right)}\right] .
\end{aligned}
$$

Thus, the phase factor $e^{i \Delta \eta(\theta)}$ is the coherent (i.e., with respect to amplitudes) average of $e^{i\left(\eta_{d}-\eta_{p}\right)}(d-p$ interference) and $e^{i\left(\eta_{s}-\eta_{p}\right)}$ (s- $p$ interference) with the relative weight

$$
\begin{aligned}
W(\theta) & =B g(\theta), \quad B=\frac{c_{d}}{c_{s}}, \\
g(\theta) & =\frac{Y_{20}(\theta, \varphi)}{Y_{00}(\theta, \varphi)}=\frac{\sqrt{5}}{4}[3 \cos (2 \theta)+1] .
\end{aligned}
$$

In other words, $\Delta \eta(\theta)$ can be regarded as a vectorial average of $\eta_{s}-\eta_{p}$ and $\eta_{d}-\eta_{p}$ with the relative weight $W(\theta)$. Equivalently, $\Delta \eta(\theta)$ may be presented as

$$
\tan \Delta \eta(\theta)=\frac{\cos \left(\eta_{s}-\eta_{p}\right)+W(\theta) \cos \left(\eta_{d}-\eta_{p}\right)}{\sin \left(\eta_{s}-\eta_{p}\right)+W(\theta) \sin \left(\eta_{d}-\eta_{p}\right)} .
$$

The energy derivative of $\Delta \eta(\theta)$ does not give us additional information about the photoionization amplitudes but provides us with the group delay and may enhance the sensitivity to the energy-dependent behavior of the twophoton ionization amplitudes, as described below.

Note two important characteristics of $\Delta \eta(\theta)$ : (i) $\Delta \eta(\theta)$ exhibits a quasicosine shape and monotonic dependence on $\theta$ due to the geometric factor $g(\theta)$ [see Figs. 2(c)-2(e) and the Appendix), and (ii) $\Delta \eta(\theta)$ is sensitive to the two-photon ionization dynamics due to the dynamical factor $B$. For example, if the two-photon pathways are close to an intermediate discrete resonance (but still well outside the bandwidth), the group delay difference $(\partial \Delta \eta / \partial \epsilon)(\theta)$ is sensitive to it through rapid change in $B$, while $d \eta_{s} / d \epsilon$, $d \eta_{p} / d \epsilon$, and $d \eta_{d} / d \epsilon$ are small individually, as can be seen in Fig. 3.
We now turn to photoionization from a $p$ orbital, which includes the present case of $\mathrm{Ne} 2 p$ ionization and is more complicated. The complexity arises from two sources. We have three incoherent contributions from $m=0$ and \pm 1 for the magnetic sublevels of the remaining ion core $\mathrm{Ne}^{+}$and four contributions of partial waves $s, p, d$, and $f$ in the photoelectron wave packet. Detailed derivations of the equations describing the PADs are given in the Appendix, and, here, we describe only the results relevant to the present discussion. For $m= \pm 1$, we have a pair of twophoton pathways via $d$ intermediate states, i.e., $p \rightarrow d \rightarrow p$ and $p \rightarrow d \rightarrow f$, together with the single-photon pathway $p \rightarrow d$. Thus, only three partial waves are involved, and, therefore, a discussion similar to that above for $s$ ionization holds.

For $m=0$, we have two-photon pathways via $s$ and $d$ intermediate states, leading to $p$ and $f$ final states, together with single-photon ionization that leads to $s$ and $d$ final states. Thus, there are four partial waves involved. Although we can derive equations similar to Eqs. (20) and (21) [see Eqs. (A14)-(A17)], what we can extract from the measurement is only a vectorial average of phase differences $\eta_{l}-\eta_{l^{\prime}}$ between even and odd different partial waves $l$ and $l^{\prime}$. We can define the angle-resolved phase difference $\Delta \eta_{m}(\theta)$ for each $m$ [see Eq. (5)], which is also a vectorial average of $\eta_{l}-\eta_{l^{\prime}}$. Similar to ionization from the $s$ state, the energy derivative of $\Delta \eta_{m}(\theta)$ may be regarded as an angle-resolved group delay between single- and twophoton wave packets for each $m$.

In the experiment, we measure an (incoherently) weighted average $\Delta \tilde{\eta}$ of angle-resolved phase differences $\Delta \eta_{m}$ of different $m$ as defined in Eqs. (5) and (6). One can introduce the energy derivative of the weighted average phase difference $\Delta \tilde{\eta}(\theta)$ and may call it generalized delay, but this definition of time delay is different from that commonly employed for the time delay of an incoherent sum of wave packets. Usually, the phase of each wave packet is first differentiated with respect to energy and then averaged over $m$ [57], while, in this study, $(d \Delta \tilde{\eta} / d \epsilon)(\theta)$ first averages the wave packet phase over $m$ and then differentiates it with respect to the photoelectron energy.

\section{SUMMARY AND OUTLOOK}

In this work, we have described a new method to determine the angle-resolved relative phase between singleand two-photon ionization amplitudes and used it to measure the $2 p$ photoionization of $\mathrm{Ne}$. Our approach allows us to explore the phase difference between different ionization pathways, e.g., those of odd and even parities, with the same photoelectron energy.

The method is based on FEL radiation, so that it can be extended to shorter wavelengths, eventually to inner shells, which lie in a wavelength region where optical lasers have reduced pulse energy. This method is an important addition to the armory of techniques available to attosecond science 
and gives access to the phase difference between single(odd-parity) and two-photon (even-parity) transition amplitudes or the energy variation of the phase of two-photon ionization amplitudes affected by the intermediate resonances, as seen in the Ne $2 p$ photoionization. For $n s^{2}$ subshells of atoms, e.g., $1 s^{2}$ of $\mathrm{He}, 1 s^{2}$ and $2 s^{2}$ of $\mathrm{Ne}$, etc., in particular, one can extract the eigenphase differences for $s, p$, and $d$ partial waves of electron-ion scattering, and their energy derivatives correspond to the Wigner delay difference of the partial waves. This method is also applicable to molecules.

While it does not yet appear to be feasible with present high-harmonic generation (HHG) sources, it may become possible in the future, but there are many technical challenges. Since HHG sources produce a frequency comb, the chief technical challenges are to filter the beam to achieve bichromatic spectral purity, maintain attosecond temporal resolution, and provide enough pulse energy at the fundamental wavelength to initiate two-photon ionization. Furthermore, HHG sources have not yet demonstrated the level of phase control which we have at our disposal. Given the rapid progress in HHG sources, these conditions may eventually be met, in which case our method will become more widely accessible.

The information obtained by this method is complementary to that of streaking and RABBITT methods, in the sense that different phase differences are measured. We have directly measured the angle-resolved average phase difference $\Delta \tilde{\eta}(\theta)$ of two-photon amplitude relative to the single-photon ionization amplitude. The basic physics giving rise to its angular dependence is related to interference between photoelectron waves emitted in one- and twophoton ionization, consisting of partial photoelectron waves with opposite parities. We have shown that the overall shape of $\Delta \tilde{\eta}(\theta)$ versus the angle can be understood qualitatively.

\section{ACKNOWLEDGMENTS}

This work was supported in part by the X-ray Free Electron Laser Utilization Research Project and the X-ray Free Electron Laser Priority Strategy Program of the Ministry of Education, Culture, Sports, Science, and Technology of Japan (MEXT) and the IMRAM program of Tohoku University, and the Dynamic Alliance for Open Innovation Bridging Human, Environment and Materials program. K. L. I. gratefully acknowledges support by the Cooperative Research Program of the Network Joint Research Center for Materials and Devices (Japan), Grantin-Aid for Scientific Research (Grants No. 16H03881, No. 17K05070, No. 18H03891, and No. 19H00869) from MEXT, JST COI (Grant No. JPMJCE1313), JST CREST (Grant No. JPMJCR15N1), MEXT Quantum Leap Flagship Program (MEXT Q-LEAP) Grant No. JPMXS0118067246, and Japan-Hungary Research Cooperative Program, JSPS and HAS.E. V.G. acknowledges the Foundation for the
Advancement of Theoretical Physics and Mathematics "BASIS." D. Y. acknowledges supports by JSPS KAKENHI Grant No. JP19J12870, and a Grant-in-Aid of Tohoku University Institute for Promoting Graduate Degree Programs Division for Interdisciplinary Advanced Research and Education. We acknowledge the support of the Alexander von Humboldt Foundation (Project Tirinto), the Italian Ministry of Research Project FIRB No. RBID08CRXK and No. PRIN 2010 ERFKXL 006, the bilateral project CNR JSPS Ultrafast science with extreme ultraviolet free electron lasers, and funding from the European Union Horizon 2020 research and innovation program under the Marie Sklodowska-Curie Grant Agreement No. 641789 MEDEA (Molecular Electron Dynamics investigated by IntensE Fields and Attosecond Pulses), G. S. acknowledges funding from the Deutsche Forschungsgemeinschaft under the Project No. 429805582. E. V. G., A. N. G., M. M. P., and E. I. S. acknowledge funding from the Russian Foundation for Basic Research (RFBR) under the Project No. 20-5212023. T. M. and M. M. acknowledge support by Deutsche Forschungsgemeinschaft Grant No. SFB925/A1. We thank the machine physicists of FERMI for making this experiment possible by their excellent work in providing high-quality FEL light.

\section{APPENDIX: PERTURBATION THEORY. DERIVATION OF EQUATIONS IN THE INDEPENDENT PARTICLE MODEL}

In addition to the approximations described in Sec. IV C (the dipole approximation, the validity of the lowest nonvanishing order perturbation theory), here, we add the $L S$-coupling approximation within the independent particle model. The photoelectron angular distribution $I(\theta ; \phi)$ of a Ne $2 p$ electron can be derived by standard methods [58] in the form

$$
I(\theta ; \phi)=I_{0} \sum_{m=0, \pm 1}\left|\sum_{\xi} C_{\xi}^{m}(\phi) Y_{\ell_{\xi} m}(\theta, \varphi)\right|^{2},
$$

where $m$ is the magnetic quantum number of the initial $2 p$ electron, $Y_{\ell m}(\theta, \varphi)$ is a spherical harmonic in the CondonShortley phase convention, and $I_{0}$ is a normalization factor irrelevant to further discussion; note that the dependence on $\varphi$ cancels out. The complex coefficients $C_{\xi}^{m}(\phi)$ depend on ionization amplitudes, and the index $\xi$ denotes the ionization path. For single-photon ionization, $\xi=\ell_{\xi}$, where $\ell_{\xi}$ is the orbital momentum of the photoelectron with possible values $\ell_{\xi}=s, d$. For two-photon ionization, $\xi=\left\{\ell_{\xi}, \ell_{\xi}^{\prime}\right\}$, where $\ell_{\xi}^{\prime}$ is the orbital momentum of the virtual intermediate state, with possible combinations $\xi=p s, p d, f d$.

After applying the Wigner-Eckart theorem [59] to factor out the dependence on the projection $m$, the coefficients $C_{\xi}^{m}(\phi)$ may be expressed as (for brevity, we omit the argument $\phi$ when writing the coefficients) 
$C_{s}^{0}=-\frac{1}{\sqrt{3}} D_{s} e^{i \phi}, \quad C_{d}^{0}=\sqrt{\frac{2}{15}} D_{d} e^{i \phi}, \quad C_{d}^{ \pm 1}=\frac{1}{\sqrt{10}} D_{d} e^{i \phi}$,

$$
\begin{array}{rlrl}
C_{p s}^{0} & =-\frac{1}{3} D_{p s}, & C_{p s}^{ \pm 1}=0, \\
C_{p d}^{0}=-\frac{2}{15} D_{p d}, & C_{p d}^{ \pm 1}=-\frac{1}{10} D_{p d}, \\
C_{f d}^{0}=\frac{\sqrt{2}}{5 \sqrt{7}} D_{f d}, & C_{f d}^{ \pm 1}=\frac{2}{5 \sqrt{21}} D_{f d} .
\end{array}
$$

Here,

$$
D_{\xi}=d_{\xi} e^{i \eta_{\xi}}
$$

are complex reduced matrix elements, independent of $m$, with magnitude $d_{\xi}=\left|D_{\xi}\right|$ and phase $\eta_{\xi}$. Note that one- (firstorder) and two-photon (second-order) matrix elements (A4), both marked by a single index $\xi$, are, respectively, proportional to the square root of intensity and to the intensity of the associated field.

Equation (A1) can be readily cast into the form (4), where

$$
\begin{aligned}
& A_{0}=\frac{I_{0}}{4 \pi} \sum_{\lambda=0,2,4} Z_{\lambda} P_{\lambda}(\cos \theta), \\
& A=\frac{I_{0}}{4 \pi} N, \\
& \cos \Delta \tilde{\eta}=N^{-1} \sum_{\lambda=1,3} \operatorname{Re} Z_{\lambda}^{\prime} P_{\lambda}(\cos \theta), \\
& \sin \Delta \tilde{\eta}=N^{-1} \sum_{\lambda=1,3} \operatorname{Im} Z_{\lambda}^{\prime} P_{\lambda}(\cos \theta), \\
& N=\left|\sum_{\lambda=1,3} Z_{\lambda}^{\prime} P_{\lambda}(\cos \theta)\right|,
\end{aligned}
$$

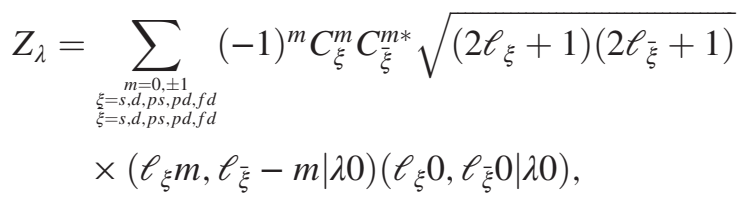

where $\left(j_{1} m_{1}, j_{2} m_{2} \mid j m\right)$ are Clebsch-Gordan coefficients [59] and $Z_{\lambda}^{\prime}=\left.Z_{\lambda}\right|_{\phi=0}$. In particular,

$$
\begin{aligned}
Z_{0}= & \frac{1}{30}\left[10\left(d_{d}^{2}+d_{s}^{2}\right)+\frac{4}{5} d_{f d}^{2}+\frac{17}{15} d_{p d}^{2}+\frac{10}{3} d_{p s}^{2}\right. \\
& \left.+\frac{8}{3} d_{p d} d_{p s} \cos \left(\eta_{p d}-\eta_{p s}\right)\right] .
\end{aligned}
$$

Equations (A2)-(A10) define $\Delta \tilde{\eta}$, provided the reduced matrix elements (A4) are calculated. The intensities of the fundamental and of the second harmonic are factored out in the coefficients $Z_{\lambda}^{\prime}$; therefore, they cancel out in Eqs. (A7) and (A8), and the phases $\Delta \tilde{\eta}$ are independent of the intensities of the harmonics.

Note that the angle-resolved average phase difference $\Delta \tilde{\eta}$ between one- and two-photon ionization implies not less than two ionization channels, which is reflected in the nonvanishing sum over channels in Eq. (A10). Therefore, $\Delta \tilde{\eta}$ and its energy derivative, or as we called it, generalized delay, is always angle dependent.

The coefficients (A10) are directly related to the anisotropy parameters $\beta_{\lambda}$ in the angular distribution of photoelectrons (A1) written in the form

$$
I(\theta ; \phi)=\frac{W_{0}}{4 \pi}\left(1+\sum_{\lambda=1}^{4} \beta_{\lambda} P_{\lambda}(\cos \theta)\right),
$$

where

$$
\beta_{\lambda}=\frac{Z_{\lambda}}{Z_{0}}
$$

and $W_{0}=I_{0} Z_{0}$. Substituting Eqs. (A2), (A3), and (A10) into (A13), one can express the anisotropy parameters in terms of reduced matrix elements (A4):

$$
\begin{aligned}
\beta_{1}= & \frac{1}{15 Z_{0}}\left[\frac{12 \sqrt{3}}{5} d_{d} d_{f d} \cos \left(\eta_{f d}-\eta_{d}-\phi\right)\right. \\
& -\frac{17 \sqrt{2}}{5} d_{d} d_{p d} \cos \left(\eta_{p d}-\eta_{d}-\phi\right) \\
& -4 \sqrt{2} d_{d} d_{p s} \cos \left(\eta_{p s}-\eta_{d}-\phi\right) \\
& +4 d_{s} d_{p d} \cos \left(\eta_{p d}-\eta_{s}-\phi\right) \\
& \left.+10 d_{s} d_{p s} \cos \left(\eta_{p s}-\eta_{s}-\phi\right)\right], \\
\beta_{2}= & \frac{1}{210 Z_{0}}\left[70 d_{d}^{2}+\frac{32}{5} d_{f d}^{2}+\frac{49}{15} d_{p d}^{2}+\frac{140}{3} d_{p s}^{2}\right. \\
- & 140 \sqrt{2} d_{d} d_{s} \cos \left(\eta_{d}-\eta_{s}\right) \\
- & \frac{48 \sqrt{6}}{5} d_{f d} d_{p d} \cos \left(\eta_{f d}-\eta_{p d}\right) \\
- & 12 \sqrt{6} d_{f d} d_{p s} \cos \left(\eta_{f d}-\eta_{p s}\right) \\
+ & \left.\frac{112}{3} d_{p d} d_{p s} \cos \left(\eta_{p d}-\eta_{p s}\right)\right],
\end{aligned}
$$




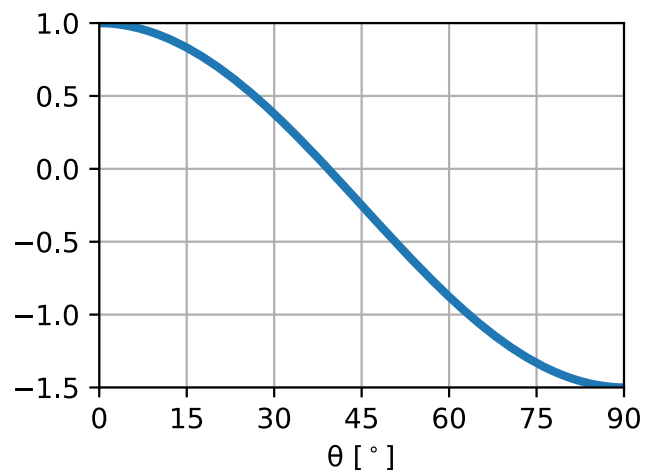

FIG. 4. Plot of $(5 \cos 2 \theta-1) / 4$ as a function of $\theta$.

$$
\begin{aligned}
\beta_{3}= & \frac{1}{30 Z_{0}}\left[\frac{16 \sqrt{3}}{5} d_{d} d_{f d} \cos \left(\eta_{f d}-\eta_{d}-\phi\right)\right. \\
& -\frac{6 \sqrt{2}}{5} d_{d} d_{p d} \cos \left(\eta_{p d}-\eta_{d}-\phi\right) \\
& -12 \sqrt{2} d_{d} d_{p s} \cos \left(\eta_{p s}-\eta_{d}-\phi\right) \\
& \left.-4 \sqrt{6} d_{s} d_{f d} \cos \left(\eta_{f d}-\eta_{s}-\phi\right)\right], \\
\beta_{4}= & \frac{4}{35 Z_{0}}\left[\frac{1}{5} d_{f d}^{2}-\frac{\sqrt{2}}{5 \sqrt{3}} d_{f d} d_{p d} \cos \left(\eta_{f d}-\eta_{p d}\right)\right. \\
- & \left.\frac{2 \sqrt{2}}{\sqrt{3}} d_{f d} d_{p s} \cos \left(\eta_{f d}-\eta_{p s}\right)\right] .
\end{aligned}
$$

The terms containing cosine functions describe the contribution from the phase difference between two-photon and single-photon ionization channels. For example, $\cos \left(\eta_{f d}-\eta_{d}-\phi\right)$ in Eq. (A14) corresponds to the phase difference between $p \rightarrow d \rightarrow f$ two-photon-ionization (TPI) and $p \rightarrow d$ single-photon-ionization (SPI) channels as well as the relative phase of the harmonics $\phi$. Note that $\beta_{2}$ and $\beta_{4}$ do not depend on $\phi$.

It immediately follows from Eqs. (A7) and (A8) that

$$
\begin{aligned}
\tan \Delta \tilde{\eta} & =\frac{\operatorname{Im} Z_{1}^{\prime} P_{1}(\cos \theta)+\operatorname{Im} Z_{3}^{\prime} P_{3}(\cos \theta)}{\operatorname{Re} Z_{1}^{\prime} P_{1}(\cos \theta)+\operatorname{Re} Z_{3}^{\prime} P_{3}(\cos \theta)} \\
& =\frac{\operatorname{Im} Z_{1}^{\prime}+f(\theta) \operatorname{Im} Z_{3}^{\prime}}{\operatorname{Re} Z_{1}^{\prime}+f(\theta) \operatorname{Re} Z_{3}^{\prime}},
\end{aligned}
$$

where the function $f(\theta)=\frac{1}{4}[5 \cos (2 \theta)-1]$ is displayed in Fig. 4. This result qualitatively explains the quasicosine shape and monotonic dependence of $\Delta \tilde{\eta}$ on $\theta$ [Figs. 2(c)-2(e)]. $Z_{\lambda}^{\prime}$ are independent of $\phi$, so that $\Delta \tilde{\eta}$ and the generalized delay are independent of the relative phase between the harmonics.

The functional form of Eq. (A18) is very general and valid, within the perturbation theory and the dipole approximation, for randomly oriented atoms and molecules, provided corresponding expressions for the coefficients $Z_{\lambda}$ in terms of the ionization amplitudes are used. Moreover, it holds for circularly polarized collinear photon beams (except for chiral targets), provided the angle $\theta$ is measured from the direction of the beam propagation.

Expression (A18) may be written in an equivalent form in terms of $\theta$-independent "average partial" TPI-SPI phase differences $\Delta \tilde{\eta}_{\lambda}(\lambda=1,3)$. Indeed, we can write Eq. (A12) explicitly in the form [see Eqs. (5) and (6)]

$$
\begin{aligned}
4 \pi W_{0}^{-1} I(\theta ; \phi)= & {\left[1+\beta_{2} P_{2}(\cos \theta)+\beta_{4} P_{4}(\cos \theta)\right] } \\
& +\left[\gamma_{1} \cos \left(\phi-\Delta \tilde{\eta}_{1}\right) P_{1}(\cos \theta)\right. \\
& \left.+\gamma_{3} \cos \left(\phi-\Delta \tilde{\eta}_{3}\right) P_{3}(\cos \theta)\right] \\
= & A_{0}(\theta)+A(\theta) \cos [\phi-\Delta \tilde{\eta}(\theta)] .
\end{aligned}
$$

Here, the prefactors $\gamma_{1}$ and $\gamma_{3}$ and phases $\Delta \tilde{\eta}_{1}$ and $\Delta \tilde{\eta}_{3}$ are independent of $\theta$ and $\phi$. It follows from Eq. (A19) that $\Delta \tilde{\eta}(\theta)$ can be viewed as a "vectorial" average of $\Delta \tilde{\eta}_{1}$ and $\Delta \tilde{\eta}_{3}$ with weights $\gamma_{1} P_{1}(\cos \theta)$ and $\gamma_{3} P_{3}(\cos \theta)$, in the sense that $\Delta \tilde{\eta}(\theta)$ is the directional angle of vector

$$
\begin{aligned}
& {\left[\gamma_{1} P_{1}(\cos \theta) \cos \Delta \tilde{\eta}_{1}\right.} \\
& \quad+\gamma_{3} P_{3}(\cos \theta) \cos \Delta \tilde{\eta}_{3}, \gamma_{1} P_{1}(\cos \theta) \sin \Delta \tilde{\eta}_{1} \\
& \left.\quad+\gamma_{3} P_{3}(\cos \theta) \sin \Delta \tilde{\eta}_{3}\right],
\end{aligned}
$$

satisfying

$\tan \Delta \tilde{\eta}(\theta)=\frac{\gamma_{1} P_{1}(\cos \theta) \sin \Delta \tilde{\eta}_{1}+\gamma_{3} P_{3}(\cos \theta) \sin \Delta \tilde{\eta}_{3}}{\gamma_{1} P_{1}(\cos \theta) \cos \Delta \tilde{\eta}_{1}+\gamma_{3} P_{3}(\cos \theta) \cos \Delta \tilde{\eta}_{3}}$.

There are simple relations between the "average partial" TPI-SPI phase differences and parameters of Eq. (A18):

$\sin \Delta \tilde{\eta}_{\lambda}=\frac{\operatorname{Im} Z_{\lambda}^{\prime}}{\left|Z_{\lambda}^{\prime}\right|}, \quad \cos \Delta \tilde{\eta}_{\lambda}=\frac{\operatorname{Re} Z_{\lambda}^{\prime}}{\left|Z_{\lambda}^{\prime}\right|}(\lambda=1,3)$

and also

$$
\gamma_{1}=\left|Z_{3}^{\prime}\right|^{-1}, \quad \gamma_{3}=\left|Z_{1}^{\prime}\right|^{-1} .
$$

As stated above, we can use the fact that the parity of Legendre polynomials obeys $P_{n}(-x)=(-1)^{n} P_{n}(x)$, so that the vector defined by Eq. (A20) changes sign upon performing the substitution $\theta \rightarrow \pi-\theta$; i.e., the two halves of the VMI image oscillate in antiphase: $\Delta \tilde{\eta}(\theta)=$ $\Delta \tilde{\eta}(\pi-\theta)+\pi$.

[1] F. Krausz and M. Ivanov, Attosecond Physics, Rev. Mod. Phys. 81, 163 (2009).

[2] E. P. Wigner, Lower Limit for the Energy Derivative of the Scattering Phase Shift, Phys. Rev. 98, 145 (1955). 
[3] S. Pabst and J. M. Dahlström, Eliminating the Dipole Phase in Attosecond Pulse Characterization Using Rydberg Wave Packets, Phys. Rev. A 94, 013411 (2016).

[4] K. Klünder, J. M. Dahlström, M. Gisselbrecht, T. Fordell, M. Swoboda, D. Guénot, P. Johnsson, J. Caillat, J. Mauritsson, A. Maquet, R. Taïeb, and A. L'Huillier, Probing SinglePhoton Ionization on the Attosecond Time Scale, Phys. Rev. Lett. 106, 143002 (2011).

[5] D. Guénot, D. Kroon, E. Balogh, E. W. Larsen, M. Kotur, M. Miranda, T. Fordell, P. Johnsson, J. Mauritsson, M. Gisselbrecht, K. Varjú, C. L. Arnold, T. Carette, A. S. Kheifets, E. Lindroth, A. L'Huillier, and J. M. Dahlström, Measurements of Relative Photoemission Time Delays in Noble Gas Atoms, J. Phys. B 47, 245602 (2014).

[6] D. Guénot, K. Klünder, C. L. Arnold, D. Kroon, J. M. Dahlström, M. Miranda, T. Fordell, M. Gisselbrecht, P. Johnsson, J. Mauritsson, E. Lindroth, A. Maquet, R. Taïeb, A. L'Huillier, and A. S. Kheifets, Photoemission-Time-Delay Measurements and Calculations Close to the 3 s-IonizationCross-Section Minimum in Ar, Phys. Rev. A 85, 053424 (2012).

[7] C. Palatchi, J. M. Dahlström, A. S. Kheifets, I. A. Ivanov, D. M. Canaday, P. Agostini, and L. F. DiMauro, Atomic Delay in Helium, Neon, Argon and Krypton, J. Phys. B 47, 245003 (2014).

[8] A. S. Kheifets, Time Delay in Valence-Shell Photoionization of Noble-Gas Atoms, Phys. Rev. A 87, 063404 (2013).

[9] S. Saha, A. Mandal, J. Jose, H. R. Varma, P. C. Deshmukh, A.S. Kheifets, V. K. Dolmatov, and S. T. Manson, Relativistic Effects in Photoionization Time Delay near the Cooper Minimum of Noble-Gas Atoms, Phys. Rev. A 90, 053406 (2014).

[10] L. Argenti, A. Jiménez-Galán, J. Caillat, R. Taïeb, A. Maquet, and F. Martín, Control of Photoemission Delay in Resonant Two-Photon Transitions, Phys. Rev. A 95, 043426 (2017).

[11] D. Busto, J. Vinbladh, S. Zhong, M. Isinger, S. Nandi, S. Maclot, P. Johnsson, M. Gisselbrecht, A. LHuillier, E. Lindroth, and J. M. Dahlström, Fanos Propensity Rule in Angle-Resolved Attosecond Pump-Probe Photoionization, Phys. Rev. Lett. 123, 133201 (2019).

[12] J. Vos, L. Cattaneo, S. L. Patchkovskii, T. Zimmermann, C. Cirelli, M. Lucchini, A. Kheifets, A. S. Landsman, and U. Keller, Orientation-Dependent Stereo Wigner Time Delay and Electron Localization in a Small Molecule, Science 360, 1326 (2018).

[13] D. Azoury, O. Kneller, M. Krüger, B. D. Bruner, O. Cohen, Y. Mairesse, and N. Dudovich, Interferometric Attosecond Lock-in Measurement of Extreme-Ultraviolet Circular Dichroism, Nat. Photonics 13, 198 (2019).

[14] J. Fuchs, N. Douguet, S. Donsa, F. Martin, J. Burgdrfer, L. Argenti, L. Cattaneo, and U. Keller, Time Delays from One-Photon Transitions in the Continuum, Optica 7, 154 (2020).

[15] M. Ossiander, F. Siegrist, V. Shirvanyan, R. Pazourek, A. Sommer, T. Latka, A. Guggenmos, S. Nagele, J. Feist, J. Burgdrfer, R. Kienberger, and M. Schultze, Attosecond Correlation Dynamics, Nat. Phys. 13, 280 (2017).

[16] M. Schultze et al., Delay in Photoemission, Science 328, 1658 (2010).
[17] P. Eckle, A. N. Pfeiffer, C. Cirelli, A. Staudte, R. Dörner, H. G. Muller, M. Büttiker, and U. Keller, Attosecond Ionization and Tunneling Delay Time Measurements in Helium, Science 322, 1525 (2008).

[18] P. Eckle, M. Smolarski, P. Schlup, J. Biegert, A. Staudte, M. Schöffler, H. G. Muller, R. Dörner, and U. Keller, Attosecond Angular Streaking, Nat. Phys. 4, 565 (2008).

[19] A. N. Pfeiffer, C. Cirelli, M. Smolarski, D. Dimitrovski, M. Abu-samha, L. B. Madsen, and U. Keller, Attoclock Reveals Natural Coordinates of the Laser-Induced Tunnelling Current Flow in Atoms, Nat. Phys. 8, 76 (2012).

[20] A. N. Pfeiffer, C. Cirelli, M. Smolarski, R. Dörner, and U. Keller, Timing the Release in Sequential Double Ionization, Nat. Phys. 7, 428 (2011).

[21] P. M. Paul, E. S. Toma, P. Breger, G. Mullot, F. Augé, P. Balcou, H. G. Muller, and P. Agostini, Observation of a Train of Attosecond Pulses from High Harmonic Generation, Science 292, 1689 (2001).

[22] V. Loriot, A. Marciniak, G. Karras, B. Schindler, G. RenoisPredelus, I. Compagnon, B. Concina, R. Brédy, G. Celep, C. Bordas, E. Constant, and F. Lépine, Angularly Resolved RABBITT Using a Second Harmonic Pulse, J. Opt. 19, 114003 (2017).

[23] D. Azoury, O. Kneller, S. Rosen, B. D. Bruner, A. Clergerie, Y. Mairesse, B. Fabre, B. Pons, N. Dudovich, and M. Krüger, Electronic Wavefunctions Probed by All-Optical Attosecond Interferometry, Nat. Photonics 13, 54 (2019).

[24] D. M. Villeneuve, P. Hockett, M. J. J. Vrakking, and H. Niikura, Coherent Imaging of an Attosecond Electron Wave Packet, Science 356, 1150 (2017).

[25] S. Donsa, N. Douguet, J. Burgdörfer, I. Březinová, and L. Argenti, Circular Holographic Ionization-Phase Meter, Phys. Rev. Lett. 123, 133203 (2019).

[26] J. M. Dahlström and E. Lindroth, Study of Attosecond Delays Using Perturbation Diagrams and Exterior Complex Scaling, J. Phys. B 47, 124012 (2014).

[27] J. Wätzel, A. S. Moskalenko, Y. Pavlyukh, and J. Berakdar, Angular Resolved Time Delay in Photoemission, J. Phys. B 48, 025602 (2015).

[28] S. Heuser, Á. Jimenez Galán, C. Cirelli, C. Marante, R. Sabbar, M. Boge, M. Lucchini, L. Gallmann, I. Ivanov, A. S. Kheifets, J. M. Dahlström, E. Lindroth, L. Argenti, F. Martín, and U. Keller, Angular Dependence of Photoemission Time Delay in Helium, Phys. Rev. A 94, 063409 (2016).

[29] A. Mandal, P. C. Deshmukh, A. S. Kheifets, V. K. Dolmatov, and S. T. Manson, Angle-Resolved Wigner Time Delay in Atomic Photoionization: The 4d Subshell of Free and Confined Xe, Phys. Rev. A 96, 053407 (2017).

[30] I. A. Ivanov and A. S. Kheifets, Angle-Dependent Time Delay in Two-Color XUV + IR Photoemission of He and Ne, Phys. Rev. A 96, 013408 (2017).

[31] A. W. Bray, F. Naseem, and A. S. Kheifets, Photoionization of Xe and Xe and $\mathrm{Xe} @ \mathrm{C}_{60}$ from the $4 d$ Shell in RABBITT Fields, Phys. Rev. A 98, 043427 (2018).

[32] S. Banerjee, P. C. Deshmukh, V. K. Dolmatov, S. T. Manson, and A.S. Kheifets, Strong Dependence of Photoionization Time Delay on Energy and Angle in the Neighborhood of Fano Resonances, Phys. Rev. A 99, 013416 (2019). 
[33] C. Cirelli et al., Anisotropic Photoemission Time Delays Close to a Fano Resonance, Nat. Commun. 9, 955 (2018).

[34] M. Shapiro and P. Brumer, Principles of the Quantum Control of Molecular Processes (Wiley-Interscience, Hoboken, NJ, 2003).

[35] K. C. Prince et al., Coherent Control with a ShortWavelength Free-Electron Laser, Nat. Photonics 10, 176 (2016).

[36] V. Lyamayev et al., A Modular End-Station for Atomic, Molecular, and Cluster Science at the Low Density Matter Beamline of FERMI@Elettra, J. Phys. B 46, 164007 (2013).

[37] C. Svetina et al., The Low Density Matter (LDM) Beamline at FERMI: Optical Layout and First Commissioning, J. Synchrotron Radiat. 22, 538 (2015).

[38] E. Allaria et al., The FERMI Free-Electron Lasers, J. Synchrotron Radiat. 22, 485 (2015).

[39] V. Dribinski, A. Ossadtchi, V. A. Mandelshtam, and H. Reisler, Reconstruction of Abel-Transformable Images: The Gaussian Basis-Set Expansion Abel Transform Method, Rev. Sci. Instrum. 73, 2634 (2002).

[40] M. Di Fraia et al., Complete Characterization of Phase and Amplitude of Bichromatic XUV Light, Phys. Rev. Lett. 123, 213904 (2019).

[41] B. Diviacco, R. Bracco, D. Millo, and M. M. Musardo, Phase Shifters for the FERMI@Elettra undulators, in Proceedings of the 2nd International Particle Accelerator Conference, San Sebastián, Spain (EPS-AG, Spain, 2011), p. 3278.

[42] L. Giannessi, E. Allaria, K. C. Prince, C. Callegari, G. Sansone, K. Ueda, T. Morishita, C. N. Liu, A. N. GrumGrzhimailo, E. V. Gryzlova, N. Douguet, and K. Bartschat, Coherent Control Schemes for the Photoionization of Neon and Helium in the Extreme Ultraviolet Spectral Region, Sci. Rep. 8, 7774 (2018).

[43] M. Zangrando, D. Cocco, C. Fava, S. Gerusina, R. Gobessi, N. Mahne, E. Mazzucco, L. Raimondi, L. Rumiz, and C. Svetina, Recent Results of PADReS, the Photon Analysis Delivery and REduction System, from the FERMI FEL Commissioning and User Operations, J. Synchotron Radiat. 22, 565 (2015).

[44] L. Raimondi et al., Microfocusing of the FERMI@Elettra FEL Beam with a KB Active Optics System: Spot Size Predictions by Application of the WISE Code, Nucl. Instrum. Methods Phys. Res., Sect. A 710, 131 (2013).

[45] T. Sato and K. L. Ishikawa, Time-Dependent CompleteActive-Space Self-Consistent-Field Method for Multielectron Dynamics in Intense Laser Fields, Phys. Rev. A 88, 023402 (2013).
[46] T. Sato, K. L. Ishikawa, I. Brezinová, F. Lackner, S. Nagele, and J. Burgdörfer, Time-Dependent Complete-ActiveSpace Self-Consistent-Field Method for Atoms: Application to High-Order Harmonic Generation, Phys. Rev. A 94, 023405 (2016).

[47] K. L. Ishikawa and K. Ueda, Competition of Resonant and Nonresonant Paths in Resonance-Enhanced TwoPhoton Single Ionization of He by an Utlrashort ExtremeUltraviolet Pulse, Phys. Rev. Lett. 108, 033003 (2012).

[48] K. L. Ishikawa and K. Ueda, Photoelectron Angular Distribution and Phase in Two-Photon Single Ionization of $H$ and He by a Femtosecond and Attosecond ExtremeUltraviolet Pulse, Appl. Sci. 3, 189 (2013).

[49] Y. Orimo, T. Sato, A. Scrinzi, and K. L. Ishikawa, Implementation of the Infinite-Range Exterior Complex Scaling to the Time-Dependent Complete-Active-Space Self-Consistent-Field Method, Phys. Rev. A 97, 023423 (2018).

[50] L. Tao and A. Scrinzi, Photo-electron Momentum Spectra from Minimal Volumes: The Time-Dependent Surface Flux Method, New J. Phys. 14, 013021 (2012).

[51] A. Scrinzi, Infinite-Range Exterior Complex Scaling as a Perfect Absorber in Time-Dependent Problems, Phys. Rev. A 81, 053845 (2010).

[52] Y. Orimo, T. Sato, and K. L. Ishikawa, Application of the Time-Dependent Surface Flux Method to the Time-Dependent Multiconfiguration Self-Consistent-Field Method, Phys. Rev. A 100, 013419 (2019).

[53] A. Messiah, Quantum Mechanics (Dover, New York, 1961).

[54] B. Gao and A.F. Starace, Variational Principle for High-Order Perturbations with Application to Multiphoton Processes for the H Atom, Phys. Rev. A 39, 4550 (1989).

[55] E. I. Staroselskaya and A. N. Grum-Grzhimailo, A Variationally Stable Method in the Problem of Two-Photon Atomic Ionization, Vestn. Mosk. Univ., Ser. 3: Fiz., Astron. 5, 45 (2015) [Moscow Univ. Phys. Bull. 70, 374 (2015)].

[56] E. V. Gryzlova, A. N. Grum-Grzhimailo, E. I. Staroselskaya, N. Douguet, and K. Bartschat, Quantum Coherent Control of the Photoelectron Angular Distribution in BichromaticField Ionization of Atomic Neon, Phys. Rev. A 97, 013420 (2018).

[57] F. T. Smith, Lifetime Matrix in Collision Theory, Phys. Rev. 118, 349 (1960).

[58] M. Y. Amusia, Atomic Photoeffect (Plenum, New York, 1990).

[59] D. A. Varshalovich, A. N. Moskalev, and V. K. Khersonskii, Quantum Theory of Angular Momentum (World Scientific, Singapore, 1988). 